\title{
Stability of multiple alignments and phylogenetic trees: an analysis of ABC-transporter proteins family Holger Wagner ${ }^{1}$, Burkhard Morgenstern ${ }^{* 2}$ and Andreas Dress ${ }^{3,4}$
}

\author{
Address: ${ }^{1}$ FSPM, Faculty of Mathematics, Bielefeld University, Postfach 100131, D-33501 Bielefeld, Germany, ${ }^{2}$ Institute of Microbiology and \\ Genetics, University of Göttingen, Goldschmidtstr. 1, D-37077 Göttingen, Germany, ${ }^{3}$ CAS-MPG Partner Institute for Computational Biology, 320 \\ Yue Yang Road, 200031 Shanghai, PR China and ${ }^{4}$ Max Planck Institute for Mathematics in the Sciences, Inselstrasse 22 - 26, D-04103 Leipzig, \\ Germany \\ Email: Holger Wagner - h_wagner@t-online.de; Burkhard Morgenstern* - bmorgen@gwdg.de; Andreas Dress - andreas@picb.ac.cn \\ * Corresponding author \\ Published: 6 November 2008 \\ Algorithms for Molecular Biology 2008, 3:15 doi:10.1186/1748-7/88-3-15 \\ This article is available from: http://www.almob.org/content/3/1/15 \\ (c) 2008 Wagner et al; licensee BioMed Central Ltd. \\ This is an Open Access article distributed under the terms of the Creative Commons Attribution License (http://creativecommons.org/licenses/by/2.0), \\ which permits unrestricted use, distribution, and reproduction in any medium, provided the original work is properly cited. \\ Received: 30 April 2008 \\ Accepted: 6 November 2008
}

\begin{abstract}
Background: Sequence-based phylogeny reconstruction is a fundamental task in Bioinformatics. Practically all methods for phylogeny reconstruction are based on multiple alignments. The quality and stability of the underlying alignments is therefore crucial for phylogenetic analysis.

Results: In this short report, we investigate alignments and alignment-based phylogenies constructed for a set of $22 \mathrm{ABC}$ transporters using CLUSTAL W and DIALIGN. Comparing the 22 "one-out phylogenies" one can obtain for this sequence set, some intrinsic phylogenetic instability is observed - even if attention is restricted to branches with high bootstrapping frequencies, the so-called safe branches. We show that this instability is caused by the fact that both, CLUSTAL W as well as DIALIGN, apparently get "confused" by sequence repeats in some of the $A B C$-transporter. To deal with such problems, two new DIALIGN options are introduced that prove helpful in our context, the "exclude-fragment" (or "xfr") and the "self-comparison" (or "sc") option.

Conclusion: "One-out strategies", known to be a useful tool for testing the stability of all sorts of data-analysis procedures, can successfully be used also in testing alignment stability. In case instabilities are observed, the sequences under consideration should be carefully checked for putative causes. In case one suspects sequence repeats to be the cause, the new "sc" option can be used to detect such repeats, and the "xfr" option can help to resolve the resulting problems.
\end{abstract}

\section{Introduction}

For more than three decades, sequence-based computer programs for phylogenetic reconstruction are routinely used for sequence-data analysis, see $[10,34,11]$ for reviews of various popular methods. They are crucial tools not only for understanding gene and species evolution, but also for analyzing the structure and function of proteins. A good example is the family of ABC-transporter proteins that became a topic of active research during the last few years (ABC stands for ATP-Binding Casette). At present, thousands of different $\mathrm{ABC}$ transporters are known; for the bacterium Sinorhizobium meliloti alone, around 150 ABC-transporter proteins have been identified $[2,4]$. Thus, the sheer size of the ABC-transporter family makes it desirable to reduce experimental costs by using in silico methods for structural and functional annotation. In view of 
some recent articles in which a close correspondence between the phylogeny and physiological function of these proteins was demonstrated (cf. $[5,15,18,19,30]$ ), phylogenetic analysis may apparently be of some use in this context: Putative functions of unknown ABC transporters can be delineated from their position within the family tree. However, this perspective of using tree-reconstruction programs for in silico annotation makes it indispensable to thoroughly check their current reliability, as well as that of the multi-alignment programs on which such reconstruction programs are based.

Herein, we study the reliability of two standard multialignment programs, CLUSTALW and DIALIGN, by applying them to $\mathrm{ABC}$ transporters. We apply the "oneout strategy" to analyze the stability of the alignments constructed by these two methods, i.e., by removing - one by one - individual sequences from the input sequence set: Ideally, removing sequences from a sequence set should not affect the alignment of the remaining sequences; their alignment should agree with the subalignment derived for them by restricting the alignment constructed for the complete sequence set (Fig. 1). But, as we will demonstrate, this stability requirement is often not fulfilled, and the resulting instabilities of alignments may even cause - and can be detected by - instabilities of the branching structure of the phylogenetic trees constructed from the alignments.

The most popular tool to test the reliability of a tree topology is the bootstrapping method. It works as follows [9,6]: for a fixed alignment of sequences, a collection of new alignments is generated by deleting some columns from the alignment at random and replacing them by other columns from that alignment, also picked at random. The tree-building algorithm is then applied to the resulting collection of new alignments. The relative frequency with which a chosen branch appears, its bootstrapping frequency, is then taken as a measure of the confidence one can have in this branch - or, more precisely, in the phylogenetic relevance of the corresponding split of the underlying sequence set induced by this branch. Hence, bootstrapping can be used to assess the reliability of a tree's branches derived from a fixed alignment and to identify
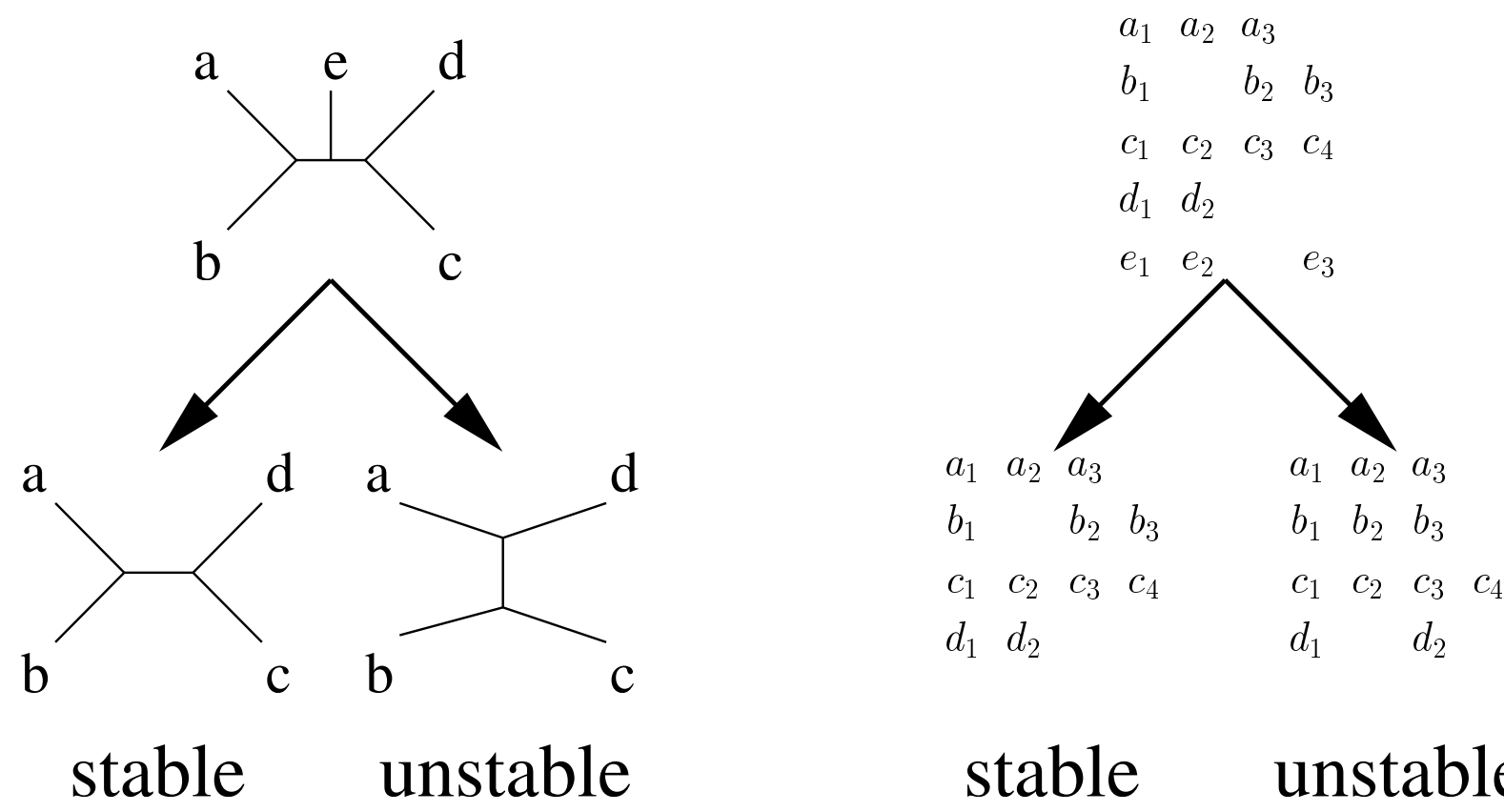

\section{stable unstable}

\section{Figure I}

Stability versus instability of molecular phylogenies (left) and alignments (right): A tree-reconstruction program produces a stable phylogeny if the phylogeny derived for a smaller sequence set coincides with the subphylogeny induced, for this smaller set, from the 'complete' phylogeny. Analogously, a multi-alignment program produces a stable alignment if the alignments of smaller sequence sets show the same columns as the corresponding subalignment of the 'complete' alignment. 
its safe branches that we define as branches with a bootstrapping frequency above a certain threshold, but it does not reflect the reliability of the alignment itself. For this paper, we used a threshold value of $5 / 6$.

In this study, only the safe branches of a tree topology will be considered when comparing trees resulting from distinct alignments, and we will confirm that, as expected, instabilities concerning these branches indicate instabilities of the underlying alignments.

Furthermore, we show that a new option that has been added in the context of our investigations to the DIALIGN software can lead to considerably improved alignments. This option, called the exclude-fragment or, for short, the $x$ fr option, allows the user to exclude certain pairs of sites, or pairs of segments, from being aligned to each other and, this way, to introduce expert knowledge into the alignment process as well as to explore interactively various alignment alternatives. In this regard, the $x f r$ option is sort of "dual" to the recently introduced anchored-alignment option where the program is required to align certain user-specified parts of the sequences to each other - provided the requirements are consistent and there is at least some degree of similarity between the specified regions $[3,27]$. In this context, we also look at the influence of the guide tree used by CLUSTAL W for constructing CLUSTAL $\mathrm{W}$ alignments.

As a by-product of our study, we found that the numerical values of the scores of multiple alignments produced by DIALIGN are sometimes noticeably below the scores of other multiple alignments that DIALIGN can be tricked to find for the same sequence set using the $x f r$ option. This observation, while perhaps disturbing, but not of immediate relevance for the user, is apparently crucial for further development of this tool. Indeed, every multi-alignment approach has to address two major problems: (a) a procedure has to be defined that, in principle, associates a score to every possible alignment of any given sequence set and (b) an optimization algorithm has to be designed that finds optimal (or near-optimal) alignments relative to that scoring procedure. Our study shows that DIALIGN can sometimes fail by some margin to find the maximum score indicating that improvements of its optimization procedures could lead to considerable improvement of the quality and, in particular, to "stabilization" of the alignments constructed by DIALIGN. Note that this result is not self-evident: According to the two parts of the alignment problem, there can be two reasons for mis-alignments as detected by instabilities: (i) the employed scoring function may itself be "unstable" (in which case it can definitely not always be in accordance with biology), and (ii) the optimization algorithm produces alignments with scores significantly below the mathematical opti- mum. Our study shows that the latter problem actually plays a non-negligible role. Thus, it makes sense to develop alternative optimisation algorithms for the fragment-based alignment approach [17,33,32].

One reason for the instabilities of the alignments constructed for the set of $\mathrm{ABC}$ transporters under investigation is the repetitive structure of three of its sequences. In a last step, we will therefore decompose these sequences into non-repetitive parts by applying another new option of DIALIGN. For the resulting new sequence set, we will also compute alignments and phylogenetic trees. Remarkably, the DIALIGN algorithm produces perfectly stable alignments and phylogenies for this new data set.

The paper is organized as follows: first, we shortly review the two multi-alignment programs CLUSTAL W [35] and DIALIGN [24,23] that we are using for our analysis. We discuss some of their weak points that proved to be relevant in our context, and we indicate possible ways of overcoming them by incorporating expert knowledge. Next, we apply CLUSTAL $\mathrm{W}$ and DIALIGN to a set of $22 \mathrm{ABC}$ transporter sequences and demonstrate that both algorithms lead to highly unstable phylogenetic trees. We show that this instability is due to instabilities of the underlying alignment procedures that are, in turn, caused by sequence repeats. Following these observations, we finally demonstrate that biologically meaningful alignments can be produced by both programs if additional information regarding these repeats or other forms of "expert knowledge" are incorporated into the alignment procedure.

\section{Alignment procedures}

In our study, we investigated CLUSTAL $\mathrm{W}$ since it is still the most popular software propgram for multi-alignment. It is a classical implementation of the progressive alignment method. That is, it reduces the computational costs of constructing a multiple alignment by reducing this task to a sequence of pairwise alignments of profiles representing previously calculated multiple alignments of subsets of the original sequence set. These pairwise alignments are calculated according to the classical Needleman-Wunsch algorithm [28] using a scoring scheme that sets rewards for pairs of matching residues against penalties imposed for gaps. Nowadays, there are more sophisticated progressive alignment methods available, for example MAFFT $[14,13]$ or MUSCLE $[8,7]$. Investigating the behaviour of these program in detail would be beyond the scope of the present study. Thus, we restrict ourselves to CLUSTAL as a prototype of the progressive alignment methods.

A crucial question is, of course, to decide in which order the pairwise alignments are computed in a progressive approach. With default options, CLUSTALW calculates, in 
a first step, all $\left(\begin{array}{l}n \\ 2\end{array}\right)$ pairwise alignments for an input set of $n$ sequences, and constructs a guide tree for the input sequences based on their pairwise similarity scores. To this end, it uses the neighbor-joining algorithm [29]. The "progressive" alignment procedure, i.e., the successive alignment of sequences and/or profiles, is then carried out according to the branching pattern of this guide tree. While this method is computationally efficient, a major point of concern is that the resulting multiple alignments - and, therefore, any phylogenetic conclusions derived from them - strongly depend on the guide tree. Clearly, any instability of the guide tree will be reflected by corresponding instabilities of the resulting alignments - in particular in the slow-and-accurate mode in which also the score function itself depends on that guide tree. Generally, CLUSTAL W produces multiple alignments of high quality if the sequences are globally related and the degree of overall similarity between them is sufficiently strong $[36,16]$.

DIALIGN uses a completely different approach. The similarity between sequences is not measured by summing up, over all sites, properly specified individual similarity scores, but rather by comparing whole sequence segments of equal length with each other. Alignments are composed from gap-free segment pairs, so-called fragment alignments or just fragments. Every possible fragment is given a score based on the probability of its random occurrence [20], and the algorithm tries to find a consistent collection of fragments with maximal total score; consistency here means the combinatorial requirement that the selected fragments are required to fit into one single output alignment and do not 'cross' each other. Gaps are not penalized in this approach - and there is, in consequence, no worrying about how to quantify gap penalties.

For pairwise alignment, the "best" chain of fragments is found using an exact recursive optimization algorithm $[21,22]$. For multi-alignments, all optimal pairwise alignments are constructed in a first step. Taking account of their individual score, fragments occurring in these pairwise alignments are then incorporated one-by-one into a growing multiple alignment in a greedy fashion, provided they are consistent with the fragments that were included previously. The nature of this approach can, of course, lead to mis-alignments, in particular for sequences that contain repeats. A single wrong fragment accepted at an early stage of the greedy procedure can subsequently prevent many correct fragments from being included into the alignment, thereby leading to an output alignment of lower over-all quality and to instability. The order in which the fragments from the optimal pairwise alignment are checked for consistency and included into the multi- ple alignment is therefore crucial for the resulting multiple alignment - somehow in analogy to (but slightly less worrying than) the problems related to the choice of the guide tree used by CLUSTAL W.

Note that CLUSTAL W and DIALIGN reflect very different philosophies regarding the definition of scores: DIALIGN uses a fixed scoring procedure whereas the scoring procedures used by CLUSTAL W depends -in the slow-and-accurate mode applied in our studies - on the guide tree used for the computation of the alignment. Thus, to compare two alignments of the same sequence set, one can compare their score if both are DIALIGN alignments whereas this would not make sense if both are CLUSTAL W alignments (and even less if one is a DIALIGN and the other one a CLUSTAL W alignment).

Two recently implemented options of DIALIGN were used in this study:

- One way of influencing the output alignment is to prevent certain pairs of sequence sites, or whole gap-free sequence segments, from being aligned with each other; we implemented this exclude-fragment (or $x f r$ ) option to improve the alignment of the ABC transporters in the present study. The $x f r$ option can be used to prevent early inclusion of misleading fragments in the greedy alignment procedure outlined above, but also to explore interactively various alignment alternatives.

- The self-comparison (or sc) option was implemented to search for repeats in a given sequence. To this end, a sequence is aligned with itself in such a way that any single sequence entry can be aligned to any other entry except itself.

\section{Sequence data and definitions 3. I Sequence data}

In this study, all investigations of ABC transporters - or, for short, ABCs - are based on the ATPase-domains of $\mathrm{ABC}$ transporters. Their primary structure is characterized by three highly conserved sequence motifs: two Walker motifs called A and B that represent the ATP-decomposing domains whereas the physiological function of a third motif, the so-called signature sequence $S$, is still unknown. In [31], these subsequences are characterized as follows:

\section{A : Gx $x$ G $x G K[S, T], \mathbf{S}: L S G G Q[Q, R, K] R, \mathbf{B}: h h h$ $h D$,}

where $x$ stands for an arbitrary, and $h$ for an arbitrary hydrophobic, amino acid. The order of these three motifs is - at least for the sequences considered in this study - 


$$
-\mathrm{A}-\mathrm{S}-\mathrm{B}-
$$

(from N- to C-terminus), with dashes representing arbitrary amino-acid sequences. Some of the ABCs comprise two sets of these motifs, so their structure is

$$
-A_{1}-S_{1}-B_{1}-A_{2}-S_{2}-B_{2}-
$$

More specifically, we will consider here a collection of 22 amino-acid sequences, i.e., the complete sequences of the following proteins: tr| Q9RK11| (S1), sp| P32010| (S2), sp| P23199| (S3), sp| P08720| (S4), sp| P31220| (S5), sp| P25885| (S6), sp| P31134| (S7), sp| P26905| (S8), sp | P30750| (S9), sp | P15031| (S10), sp | P07109| (S11), sp| P09833| (S12), sp | P16678| (S13), sp| P30963| (S14), sp| P23888| (S15), sp| P29018| (S16), sp| P27299| (S17), sp | P33116| (S18), sp| P33951| (S19), sm| b20111| (S20), sm| b21260| (S21), sm| b20141| (S22). Here, tr, sp, and sm denote entries in TrEMBL, Swissprot http:// ca.expasy.org/, and in http://sequence.toulouse.inra.fr/ rhime/Complete/doc/Complete, respectively.

\subsection{Some Definitions}

Let $X$ denote the set of all taxa under consideration, and let $T$ denote a phylogenetic tree - or, for short, a phylogeny - for $X$. Then, each branch $b$ of this tree induces a split $S(b)$ of $X$, i.e., a bipartition of $X$ into two disjoint non-empty subsets of $X$ whose union is all of $X$ while $S(b) \neq S\left(b^{\prime}\right)$ holds for any two distinct branches $b, b^{\prime}$ of $T$ (cf. e.g. [1]). Thus, each phylogeny $T$ with $N$ branches $b_{1}, \ldots, b_{N}$ gives rise to a collection $\Sigma(T):\left\{S\left(b_{i}\right): 1 \leq i \leq N\right\}$ of $N$ distinct splits of $X$ that, in turn, are well known to determine $T$ (up to canonical isomorphism). Accordingly, if bootstrapping frequencies $f(b)$ have been computed for every branch $b$ of $T$, the set of all safe branches (i.e., of all branches $b$ with a bootstrapping frequencies $f(b)$ at least $5 / 6)$ can be identified with the set of splits $\Sigma_{\text {safe }}(T):\left\{S\left(b_{i}\right): 1 \leq i \leq N, f\left(b_{i}\right) \geq\right.$ $5 / 6\}$.

Two phylogenies $T_{1}$ and $T_{2}$ of $X$ are said to be essentially concordant if each one "contains" the safe branches of the other, i.e., if

$$
\Sigma_{\text {safe }}\left(T_{1}\right) \subseteq S\left(T_{2}\right) \text { and } \Sigma_{\text {safe }}\left(T_{2}\right) \subseteq S\left(T_{1}\right)
$$

holds. Otherwise, $T_{1}$ and $T_{2}$ are called definitively distinct.

Let us now assume that we are given

- a collection $X$ of (phylogenetically related) sequences and

- an algorithm Alg for computing phylogenies from sequences.
For each subset $\tilde{X}$ of $X$, let $\operatorname{Alg}(\tilde{X})$ denote the phylogeny computed for $\tilde{X}$ using the algorithm Alg. Then, Alg is said to produce a stable phylogeny for $X$ if

$$
\Sigma_{\text {safe }}(\operatorname{Alg}(\tilde{X})) \subseteq \tilde{X} \cap \Sigma(\operatorname{Alg}(X)) \text { and } \tilde{X} \cap \Sigma_{\text {safe }}(\operatorname{Alg}(X)) \subseteq \Sigma(\operatorname{Alg}(\tilde{X}))
$$

holds for all subsets $\tilde{X}$ of $X$ of co-cardinality 1 (i.e., with\# $(X-\tilde{X})=1$ ) where, for every subset $\tilde{X}$ of $X$ and every collection of $\Sigma$ of splits of $X$, we denote by $\tilde{X} \cap \Sigma$ the corresponding collection of splits of $\tilde{X}$ defined by

$$
\tilde{X} \cap \Sigma:=\{\{Y \cap \tilde{X}, Z \cap \tilde{X}\}:\{Y, Z\} \in \Sigma, Y \cap \tilde{X}, Z \cap \tilde{X} \neq \varnothing\}
$$

\section{Results}

\section{I Molecular phylogenies}

The sequence family as aligned using CLUSTAL Wand DIALIGN. DIALIGN was used with default parameters and CLUSTAL W was run with the slow-and-accurate option. Subsequently, phylogenies were computed from the alignments using the neighbor-joining algorithm with the bootstrapping option of CLUSTAL W (1000 trials, seed of 111). This resulted in two definitively distinct phylogenies depicted in Fig. 2 ( $T_{1}$ for CLUSTAL $\mathrm{W}$ and $T_{2}$ for DIALIGN).

To investigate the stability of these phylogenies, we also computed phylogenies for the 22 "reduced" sequence families ${ }_{i}$ consisting of all sequences in except the sequence $S i(i=1, \ldots, 22)$ in the same fashion. Each of these phylogenies was essentially concordant with (the restriction to the respective sequence sets of) one of the trees shown in Fig. 2 and 3. More specifically, the CLUSTAL W alignments of ${ }_{8}, 10$, and ${ }_{22}$ led to Topology III trees (cf. Fig. 3) whereas the CLUSTAL W alignments of all other reduced sequence families led to Topology I trees. The DIALIGN alignments resulted in Topology III trees for ${ }_{5}$ and ${ }_{22}$, Topology IV trees for ${ }_{1,8}, 20$, and ${ }_{21}$, and Topology II trees for all other reduced sequence families. Hence, CLUSTAL $W$ and DIALIGN produced not only definitively distinct, but also highly unstable phylogenies for our sequence families.

\subsection{Underlying alignments}

To investigate the reasons for the occurrence of these rather distinct tree topologies, we analysed the underlying alignments. In doing so, we classified these alignments by the relative positions of the motifs $A, S$, and $\mathbf{B}$. This classification led to the 5 alignment classes $1 \mathrm{a}, 1 \mathrm{~b}, 2,3$, and 4 shown in Fig. 4 one of which, class 1a, was observed only once, viz., for ${ }_{14}$. Among these alignments, alignments in class 3 look biologically most meaningful since it implies only one duplication event. By contrast, alignment in class $1 \mathrm{a}, 1 \mathrm{~b}, 2$ and 4 would require at least two independ- 

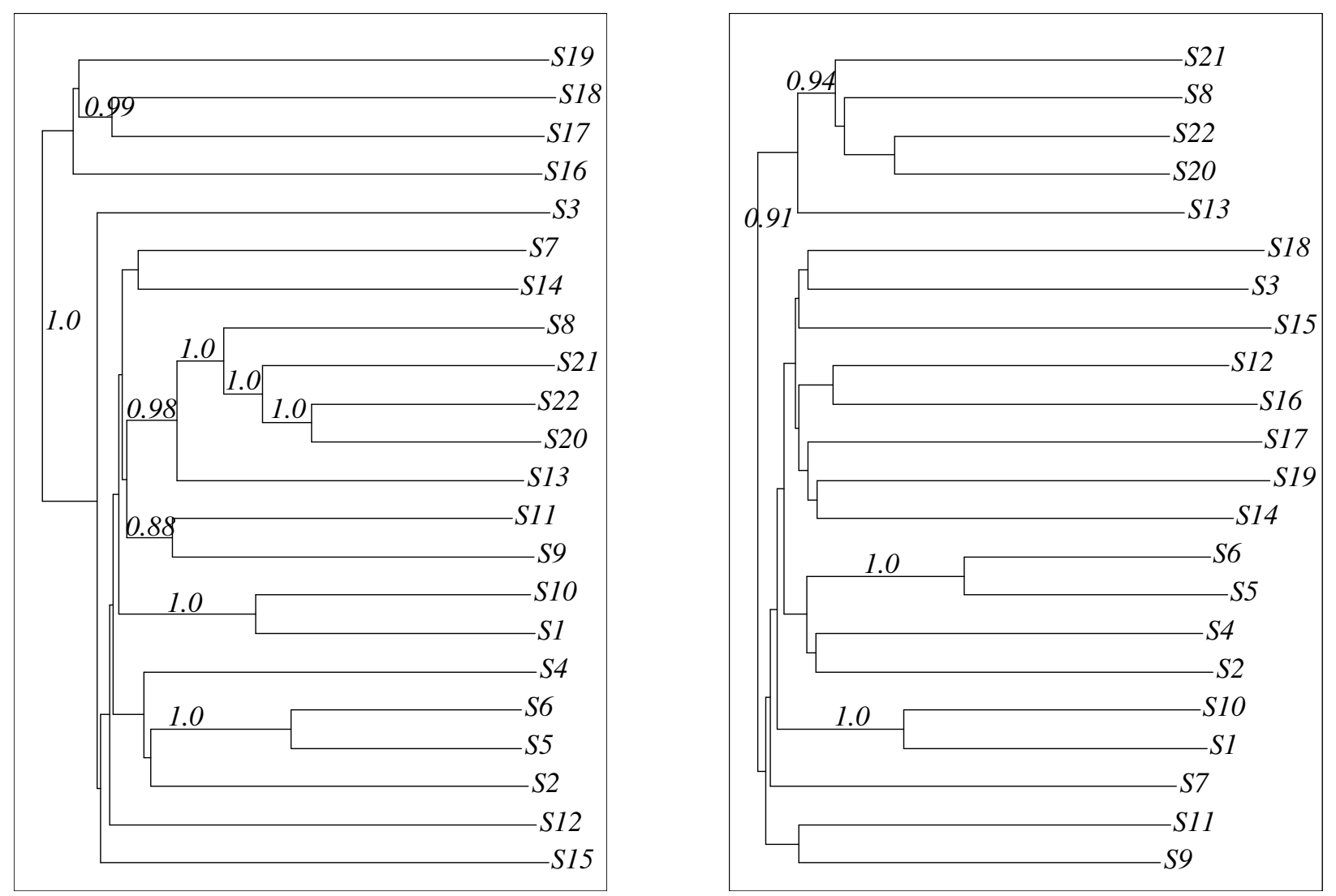

Figure 2

Two tree topologies: The tree $T_{1}$ (left) representing - up to unsafe branches - the "Topology I trees" was computed for our sequence family using CLUSTALW, and $T_{2}$ (right) representing - up to unsafe branches - the "Topology II trees" was computed using DIALIGN. The bootstrapping frequencies are shown for the safe branches of each tree. Note that the safe branches in $T_{1}$ separating $\{S I 7, S I 8\},\{S I 6, S 17, S I 8, S 19\}$, and $\{S 20, S 2 I, S 22\}$, respectively, from the sequences disapper in $T_{2}$.

ent duplication or deletion events. A meaningful alternative, namely alignments of class 5, did occur only after applying the $x f r$ option of DIALIGN for excluding class-3 type alignments.

Next, we found that alignments of class $1 \mathrm{a}$ and $1 \mathrm{~b}$ always led to Topology I trees. Analogously, alignments in the classes 2, 3, and 4 always yielded Topology II, III, and IV trees, respectively. This showed clearly that the instabilities and inconsistencies of the safe branches can be traced back to the underlying alignments.

\subsection{Modified alignments}

In this subsection, we discuss which of the 5 alignment classes have the best DIALIGN score. To compute DIALIGN scores for all alignment classes, the $x f r$ option described in 2 was used: We excluded the possibility of aligning the subwords $\mathbf{S}_{1}$ and $\mathbf{B}_{1}$ of $S 22$ with subwords of any of the other sequences aligned with these subwords in the class- 2 or class- 4 alignments. This led, for all 23 sequence sets , $1, \ldots, 22$, to alignments of class 3. Moreover, the scores of these class-3 alignments (7933-9367) were between 140 and 559 "DIALIGN units" higher than the scores of the corresponding class- 2 or class- 4 alignments. In view of the fact that the DIALIGN scores are computed in terms of the logarithm of probabilities [24], these differences are quite substantial.

Analogously, the $x f r$ option of DIALIGN was used to exclude all fragments of class- 2 and class- 4 alignments that involve the subwords $\mathbf{S}_{2}$ or $\mathbf{B}_{2}$ of the sequences $S 20$ or S21. As expected, this led to an alignment of class 5 (cf. Fig. 4) whose score 9230 was 137 "DIALIGN units" smaller than the score of the corresponding class-3 align- 

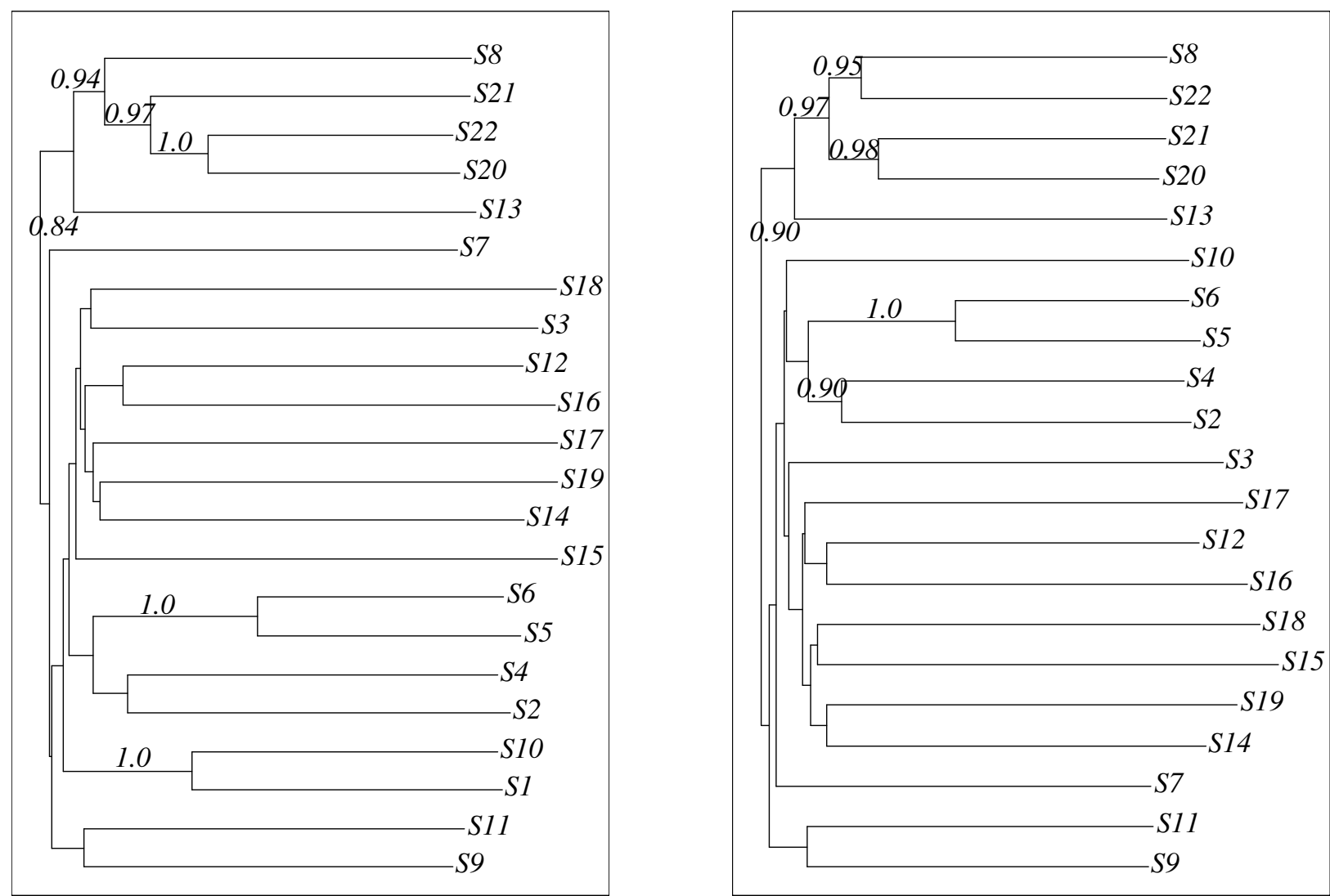

Figure 3

Representatives of "Topology III trees" (left) and "Topology IV trees" (right): Both are derived by DIALIGN. The left phylogeny is computed for using the xfr option (cf. 2 and 4.3) whereas the right one resulted from applying DIALIGN to,$:\{S 2, \ldots, S 22\}$.

ment. So, class-5 alignments appear to represent suboptimal alignments with respect to the DIALIGN score.

To investigate the influence of the guide tree on CLUSTAL W alignments, we proceeded as follows: As mentioned above, the CLUSTAL W alignment of ${ }_{10}$ led to an alignment of class 3 . In the guide tree of this alignment, we systematically replaced, quite crudely, $S i$ by $S 10$ and used this new guide tree for a CLUSTAL W alignment of ${ }_{i}$.

In each case, this resulted in an alignment of class 3 . This is quite surprising since the two guide trees leading to these rather different alignments, do not look too different. In Fig. 5, 10 out of 20 internal nodes are identical for both trees i.e. the corresponding sub-clades coincide precisely. In particular, all guide trees include a branch with taxa $S 16-S 19$, i.e., the problematic sequences in the alignments of class $1 \mathrm{a}$ and $1 \mathrm{~b}$ (cf. Fig. 4). Thus, our investigations demonstrate that small modifications of the guide tree can change the alignment significantly and that inappropriate guide trees can result in biologically unacceptable alignments. It is worth noting that all alignments of class 3 or 5 led to Topology III trees.

\subsection{Decomposition in $\mathbf{N}$ - and C-termini}

Our analyses showed that one reason for the observed instabilities is the repetitive structure of the last three sequences S20-22. Accordingly, we constructed two sequences out of each of these three sequences, each new sequence containing a single - $\mathbf{A}-\mathbf{S}$ - B- motif. To this end, we aligned each of these three sequences to itself by applying the sc option of DIALIGN to identify, and later to excise either one of, their repeats. This way, we created a new sequence family ' consisting of sequences $S 1-S 19$ together with six new sequences S20N, S21N, S22N, $S 20 C, S 21 C$, and S22C containing either the left-hand, but not the right-hand, or the right-hand, but not the lefthand, repeats of the respective sequences. 


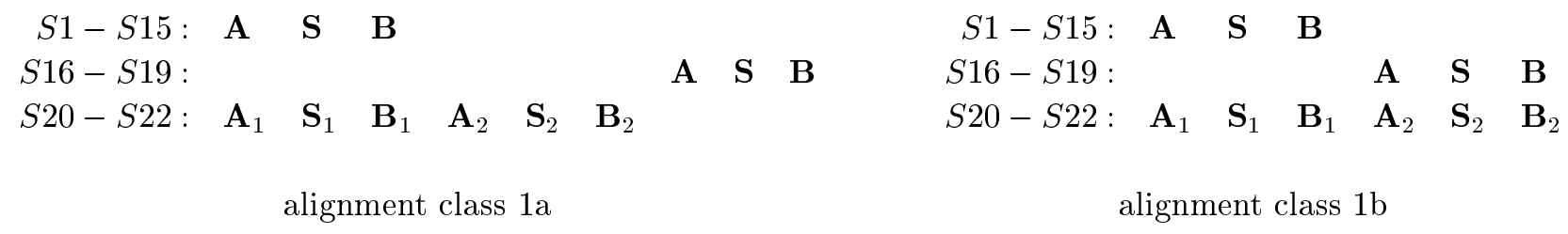

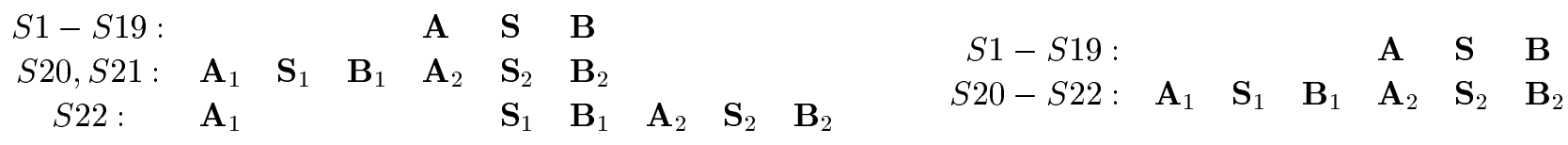

alignment class $2 \quad$ alignment class 3

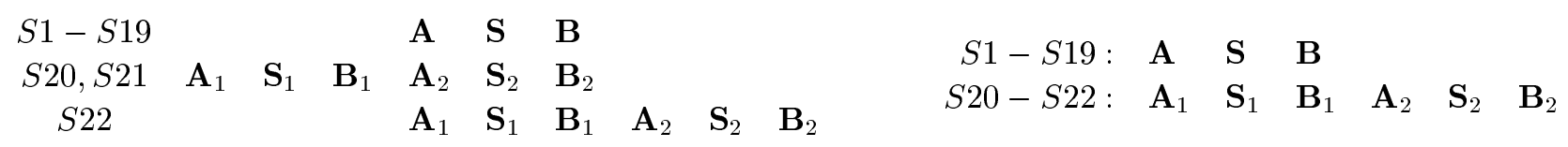

alignment class 4

alignment class 5

\section{Figure 4}

For most alignment algorithms, sequence duplications can lead to serious mis-alignments and to instabilities under minor modifications of the input sequence sets. For our original sequence set $=\{S I, \ldots, S 22\}$ and the 'reduced' sequence sets ${ }_{i}:=\backslash\{S i\}$, we obtained six classes of multi-alignments. Here, class 3 and 5 seem to be the only types of alignments that are biologically meaningful. Alignments of class 5 were obtained only once, namely by DIALIGN using the xfr option as described in 2 and 4.3. We used this option to prevent the program from aligning the motifs $A_{2}, S_{2}$ and $B_{2}$ in the sequences $S 20$ $-\mathrm{S} 22$ to the motifs $A, S$ and $B$ in the remaining sequences.

We investigated the phylogenies and alignments for the new sequence set in the same fashion as for the original sequence family. Fig. 6 shows the phylogenies derived from the CLUSTAL $\mathrm{W}$ resp. DIALIGN alignment. While the latter produced a perfectly stable phylogeny, CLUSTAL W produced only an "almost stable" phylogeny - only the branch corresponding to the split of ' into $\{S 3, S 16, S 17$, $S 18, S 19\}$ and its complement was found to be safe, yet it did not occur in any tree constructed for a proper subset of '.

The DIALIGN alignment was also shown to be stable (see Fig. 7) whereas a variety of different alignment classes showed up in the CLUSTAL W alignments, one example being also shown in Fig. 7. But there was one common feature of the CLUSTAL W alignments: The A, S, and B motifs of $S 16$ - $S 19$ were never aligned to the corresponding motifs of the other sequences.

Note that the restrictions of all CLUSTAL $W$ and DIALIGN alignments to the sequences $S 1-S 19$ coincide, respectively, with the corresponding restrictions of the align- ments of class 1 or class 3 (see Fig. 4). Thus, it is no surprise that the restrictions of the trees of ' to the taxa $S 1$ to $S 19$ also coincide with the corresponding restrictions of the Topology I (CLUSTAL W) and Topology III (DIALIGN) trees shown in Fig. 2 and 3.

This demonstrates that the two different approaches towards improving the DIALIGN alignment, namely the exclusion of fragments and the decomposition of the repetitive sequences in $\mathrm{N}$ - and $\mathrm{C}$-termini, do not lead to contradictory trees.

Furthermore, these analyses of the CLUSTAL W alignments and the trees derived from them explain in particular the stability of the branch containing the four taxa $S 16$ to $S 19$. And they show that not all the problems concerning the CLUSTALW alignments of the ABCs are caused by repeats. Additionally, we analyzed the alignments and the trees of the sequence set ": $=\{S 1, \ldots, S 19\}$ : Again DIALIGN produced a stable alignment and a stable phylogeny that coincided with the restriction of a class-3 alignment and a Topology III tree to this subset, respectively, while 

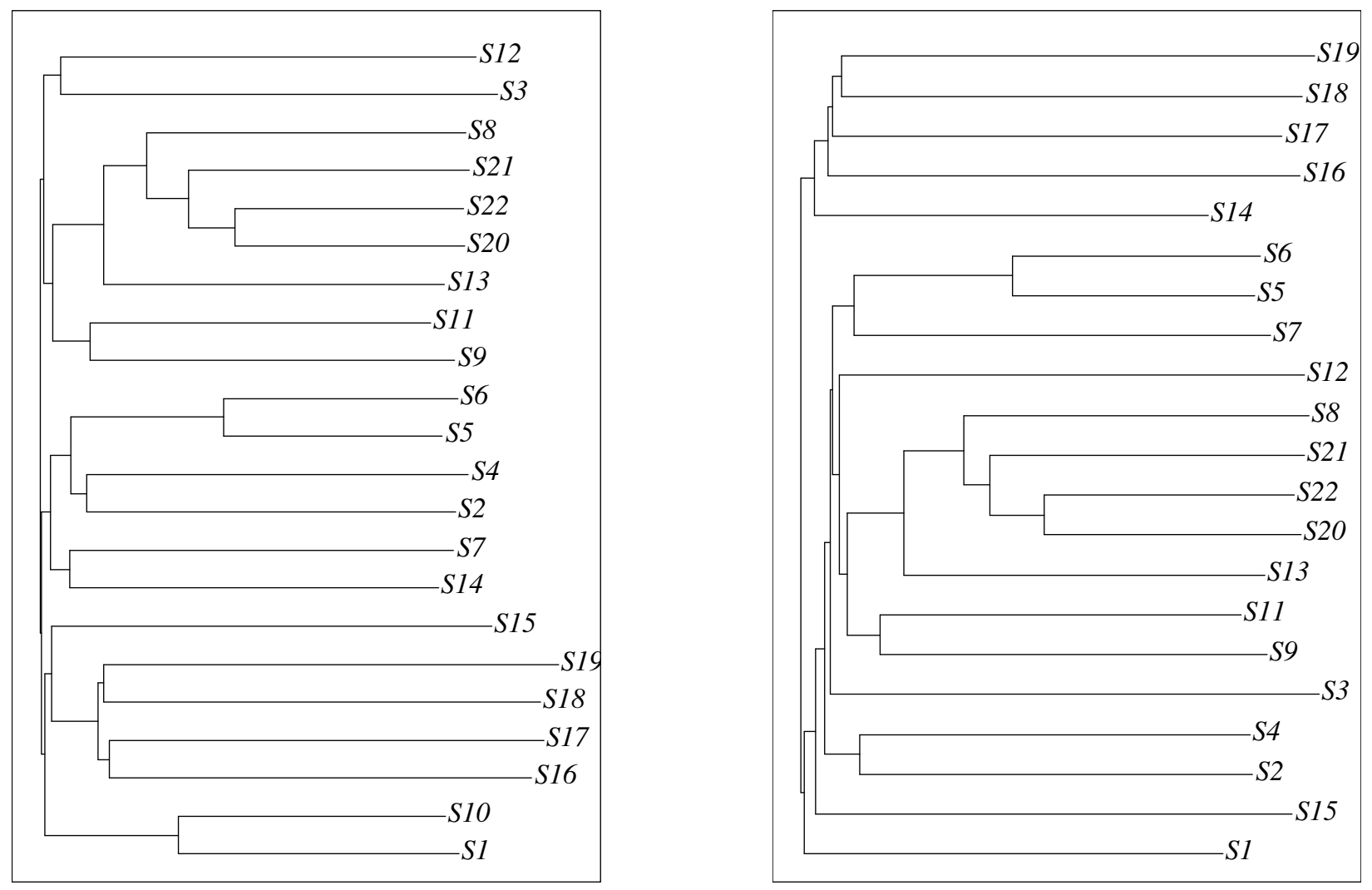

\section{Figure 5}

The guide trees of the CLUSTAL $\mathbf{W}$ alignments of (left) and of ${ }_{10}$ (right). The left guide tree resulted also from an alignment of class ( $\mathrm{Ib}$ ), the right one also from an alignment of class 3 . Note that both exhibit a branch that separates the sequences SI6-SI9 from the remaining sequences.

the alignment as well as the phylogeny produced by CLUSTAL W still turned out to be unstable: The tree produced by CLUSTAL W for " coincides with the restriction of the tree derived for '. But the sequence set consisting of $S 1-S 6$ and $S 8-S 19$, for example, leads to an alignment of class 3 and a Topology III tree.

\subsection{Relation between branches and function}

So far, we emphasized differences between tree topologies and the problems concerning their stability. In this section, we discuss which branches all our trees have in common and whether they are related to the physiological functions of the ABCs.

All six tree topologies separate the following sequence clusters from the remaining sequences (often by safe branches): $\{S 1, S 10\},\{S 2, S 4\},\{S 5, S 6\},\{S 9, S 11\},\{S 8$, $S 13, S 20, S 21, S 22\},\{S 8, S 20, S 21, S 22\},\{S 20, S 22\}$.
Furthermore, replacing each of the sequences S20, S21, $S 22$ by the corresponding $\mathrm{N}$ - and C-terminal sequences S20N, S21N, S22N and S20C, S21C, S22C, the same holds for the two trees constructed for ' which, in addition, separate $\{S 8, S 20 N, S 21 N, S 22 N\}$ suggesting a a slower evolution of the N-terminal motifs of the sequences S20,S21, S22 compared with the evolution of the C-terminal motifs of these sequences.

The splits mentioned above are related to the physiological function of the ABCs: $S 1$ and $S 10$ are iron transporters of Streptomyces coelicolor (S1) and E. coli (S10), S2 and S4 are related to the daunorubizin resistance of Streptomyces peucetius (S2) and Rhizobium leguminosum (S4), S9 and $S 11$ transport amino acids in E. coli and $S 5$ and $S 6$ are ABCs from the MURA-RPON intergenic region of E. coli and from the NTRA/RPON 5 ' region of Rhizobium meliloti.

Finally, $S 13$ is a phosphonates transporter of E. coli, whereas $S 8, S 20, S 21$, and $S 22$ are transporter of peptides: 

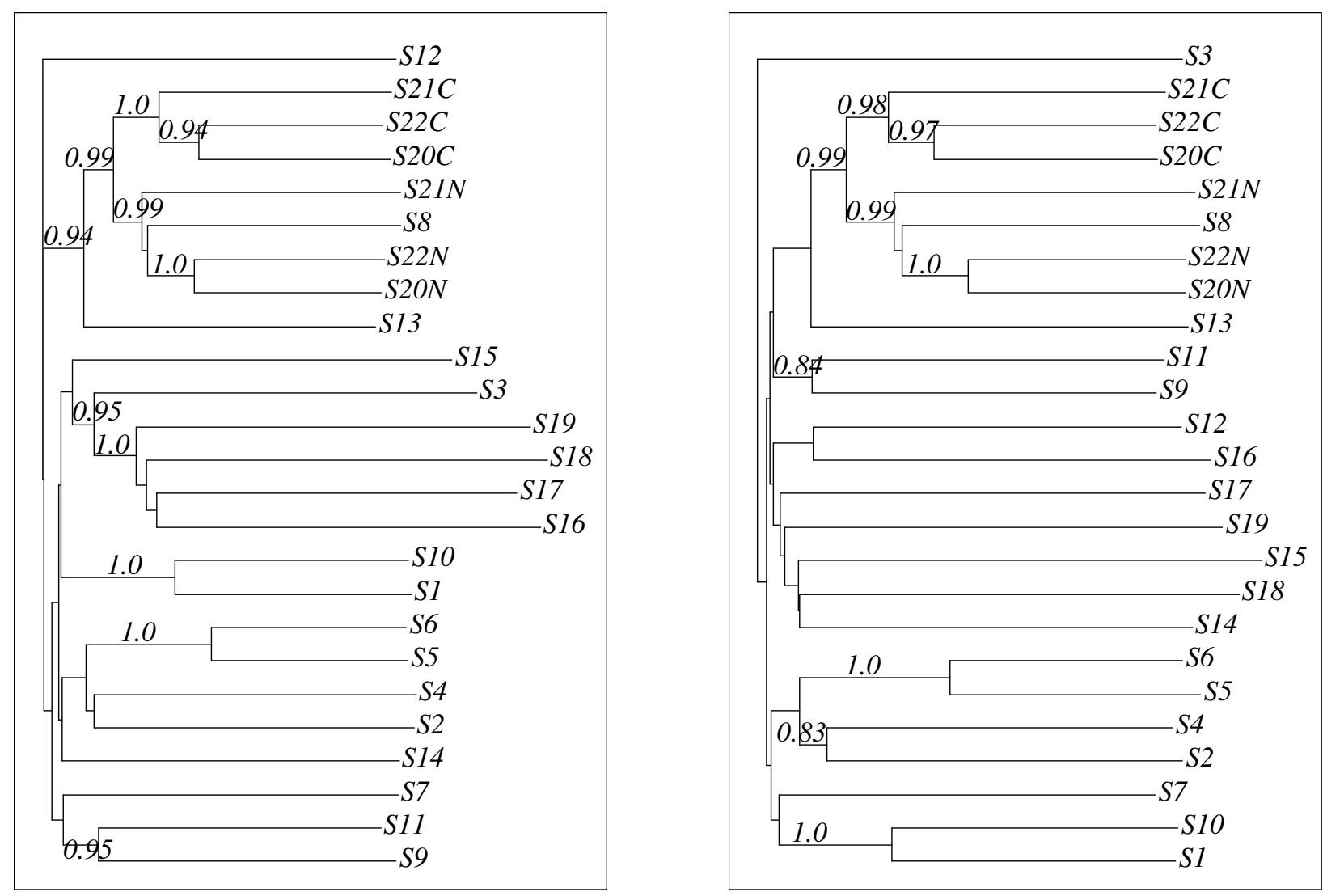

Figure 6

Phylogenies for the sequence set '. On the left (right) hand side, one finds the phylogeny derived from the CLUSTAL W (DIALIGN) alignment.

S8 is a dipeptide transporter of Bacillus subtilis, whereas S20, S21, and S22 are oligopeptide transporters of Sinorhizobium meliloti. Furthermore, the trees constructed for ' suggest that the common precursor of these five sequences differentiated into the phosphonates transporter $S 13$ and the common precursor of the four peptide transporters $S 8$,
S20, S21, and S22. They also suggest that this precursor differentiated further into the dipeptide transporter $S 8$ and the common precursor of the three oligopeptide transporters $S 20, S 21$, and $S 22$ that evolved from this precursor by motif duplication which, in turn, differentiated further into $S 21$ and the common precursor of $S 20$ and

$$
\begin{aligned}
& S 1-S 15: \quad \text { A } \quad \text { S } \mathbf{B} \\
& S 16-S 19: \quad \mathbf{A} \quad \mathbf{S} \mathbf{B} \\
& S 1-S 15: \quad \mathbf{A} \quad \mathbf{S} \quad \mathbf{B} \\
& S 16-S 19 \text { : } \\
& \text { A } \mathrm{S} \quad \mathrm{B} \\
& S 20 \_N / C-S 22_{-} N / C: \quad \mathbf{A} \quad \mathbf{S} \quad \mathbf{B} \\
& S 20 \_N / C-S 22_{-} N / C: \text { A } \quad \text { S B }
\end{aligned}
$$

\section{DIALIGN}

\section{CLUSTAL W}

\section{Figure 7}

Alignments for '. The DIALIGN alignment (left) was found to be stable. The other alignment is only one example of a variety of different alignments obtained for this sequence set and its subsets. 
S22 showing a stronger conservation of their N-terminus copy of the - A - S - B - motif than of their C-terminus copy. These parts of the sequences apparently were allowed to adapt to the new function more freely, suggesting that, after all, the class- 5 alignments might, in spite of their lower DIALIGN score, be more appropriate than the class-3 alignments.

\section{Summary and discussion}

Phylogenetic tree reconstruction from sequence data can be a very difficult task that involves two major subproblems, namely $(a)$ to construct a multiple alignment of the input sequences in such a way that biologically related parts of these sequences are assigned to each other and (b) to construct a phylogenetic tree based on such an alignment. If these subtasks are to be solved algorithmically, two types of questions are to be addressed: suitable scoring functions need to be defined assigning quantitative confidence scores to each possible multi-alignment or phylogenetic tree, respectively. These scoring functions should be defined in such a way that biologically reasonable alignments or trees are more likely to receiving higher scores than biologically meaningless ones. Given such a scoring scheme, the second problem is an optimization problem, namely to find alignments or trees, respectively, with optimal (or near-optimal) scores. Thus, phylogenetic tree reconstruction can be affected by two different types of errors. One kind of error reflects modeling problems such as unsuitable definitions of alignment scores or (dis)similarities for tree reconstruction. The other kind of errors are due to problems with finding the optimal alignment for a fixed alignment score and the optimal tree for a fixed (dis)similarity matrix. Both errors can, of course, produce instable alignments and phylogenies.

In this study, we restricted our attention to so-called safe branches of the phylogenies under investigation, i.e. to branches with bootstrap values above some threshold value. In view of their rather restrictive definition, it was natural to guess that that instabilities of safe branches indicate instabilities of the underlying alignments. And, indeed, we could relate distinct types of tree topologies to distinct alignment classes which were defined in terms of the relative position of the $-\mathbf{A}-\mathbf{S}-\mathbf{B}-$ motifs. Obviously, the threshold value of $5 / 6$ that we used in our definition of safe branches is somewhat arbitrary. But, for the sequence set considered in this study, it worked well: the different types of tree topologies that we observed could be related to distinct classes of sequence alignments. For smaller threshold values, it is quite likely that more safe branches will lead to more tree-topology types demanding the consideration of more subtle differences between the corresponding alignments.
Furthermore, we could show that preventing certain pairs of sequence sites, or whole gap-free sequence segments, from being incorporated into a DIALIGN alignment by using the $x f r$ option, resulted in the perfectly stable and biologically meaningful class-3 alignment which, in addition, had always a higher score than the other alignments. It is worth noting that this is no proof that the class- 3 alignment represents the optimal alignment of our sequence set with respect to the DIALIGN score - there could be distinct alignments with even higher scores - and even less that it is the "biologically correct" alignment. But it allowed us to avoid alignments that are obviously suboptimal, and we could show that these class- 3 alignments produce stable tree topologies. For lack of appropriate scoring parameters, a similar analysis could not be performed for CLUSTAL W. But we could demonstrate that small variations of the guide tree can result in rather different alignments and definitively distinct tree topologies. This might be a good explanation for the instabilities produced by CLUSTAL W for our sequence set.

The principal reason for the observed instabilities is probably the repetitive structure of some of the sequences under consideration. It is well known that repeats cause serious problems for all alignment algorithms [12]. Here, both subtasks in sequence alignment are concerned: it is possible that, due to repeats, biologically meaningful alignments receive lower scores than alternative, yet biologically unreasonable alignments. In addition, repeats can prevent optimization algorithms from finding (near)optimal alignments. Both types of errors have been observed, for example, for the hox-gene cluster, see [26] for more details.

In this study, we obtained stable tree topologies after constructing new repeat-free sequences from our sequences using the sc option of DIALIGN. Using these new sequences, DIALIGN produced stable alignments whereas CLUSTAL W still produced unstable alignments, yet essentially stable phylogenies. An explanation for this observation (that unstable alignments can result in stable phylogenies) is perhaps that the threshold value 5/6 in the definition of safe branches is too high to reflect instabilities of the trees. Hence, in this case, one should actually use a lower threshold value, redo all analyses, and check whether such a refinement allows to find instabilities of the trees and to trace them back to the observed alignment instabilities. In our study, only a small set of ABCs was analysed. But, as will be shown in a forthcoming article (H. Wagner and N. Diaz, in preparation), analogous analyses are also required in the phylogenetic investigation of larger sets of $\mathrm{ABCs}$ - in particular, since quite a few $\mathrm{ABCs}$ have repetitive structure. Note that complete ABCs are composed of three kinds of domains, the ATPases, the transmembrane domains, and the periplasmatic binding 
domain. In the present study, we only considered repeats within the ATPases (see Section 3.1), but repeats can also occur in other domains.

Note that the requirement of stability is only a necessary but not a sufficient condition for the reliability of the output of an alignment or tree-reconstruction program. It does not guarantee its "correctness", and the same holds, of course, for score-optimal results: Even if one could show that a certain alignment has optimal score, that would not imply that it is also biologically correct since the underlying scoring function may assign optimal numerical scores to biologically wrong alignments. In general, a (near-)optimal alignment score only implies that the corresponding alignment might be a noteworthy approximation to biological reality. In fact, it is not possible to define scoring functions for sequence alignment in such a way that score-optimal alignments necessarily coincide with biologically correct ones. For real-world sequences, common ancestry and common function and structure do not always correspond to similarity at the primary-sequence level. This is a fundamental limitation for all automated alignment approaches. To overcome these limitations, semi-automatic alignment methods have been proposed where expert knowledge can be used to guide the alignment procedure. The exclude fragments $(x f r)$ option introduced in this article offers one way to support such semi-automatic approaches. An even stronger option is the previously introduced anchored alignment option where the program is forced to align certain user-defined parts of the sequences to each other, see for example [27].

Stability analysis in phylogenetic studies is helpful to learn in more detail which alignments and (dis)similarities may be better descriptions of various aspects of sequence evolution. E.g., if phylogenetic analyses with different tools consistently lead to the same stable results, then there is at least a good chance that these results reflect the correct branching pattern of the evolution of the sequences under consideration. For similar reasons, we recently introduced a tool that compares distinct multialignments of the same sequence data set [25]. Such coincidences between safe branches of different trees were investigated in Section 4.5. These safe branches were shown to separate ABCs with identical function from the rest where the function of an $\mathrm{ABC}$ was defined by the transported substrate. Thus, it can be assumed that such branches of phylogenetic trees can indeed be used for functional predictions as discussed in the introduction.

Note that, in our analyses, ABCs from different organisms with identical functions were grouped together, but not ABCs from the same organism with different functions. As noted already in [30], this suggests that the segregation of the ABCs according to their function is older than their segregation according to the evolution of their respective organisms. Additionally or alternatively, horizontal gene transfer - well established in the bacterial world - could also contribute to this phenomenon.

From our analyses, we may also postulate that the phosphonate transporters and the dipeptide and oligopeptides transporters have a common ancestor; yet, in contrast to the dipeptide transporters, the oligopepetide transporters have a repetitive structure. However, the data set here is too small to unravel their history in detail. In a forthcoming article, these aspects of sequence evolution will be considered in more detail (Wagner and Diaz, in preparation).

\section{Acknowledgements}

This work was supported by the DFG via its grant MO I048/I-I to BM, its grant DR 40/I7-I to AD, by the FSPM and the International NRW Graduate School in Bioinformatics and Genome Research at Bielefeld University, and by the MPI for Mathematics in the Sciences in Leipzig. AD was supported by the Chinese Academy of Sciences and the German federal ministry of education and research (BMBF).

\section{References}

I. Bandelt $\mathrm{H}-\mathrm{J}$, Dress A: A canonical decomposition theory for metrics on a finite set. Advances Math 1992, 92:47-105.

2. Barnett $M$ : The composite genome of the legume symbiont sinorhizobium meliloti. Science 200I, 293:668-672.

3. Brudno M, Steinkamp R, Morgenstern B: The CHAOS/DIALIGN WWW server for multiple alignment of genomic sequences. Nucleic Acids Research 2004, 32:W4I-W44.

4. Capela D: Nucleotide sequence and predicted functions of the entire sinorhizobium meliloti pSymA megaplasmid. Proc Natl Acad Sci USA 200I, 98:9883-9888.

5. Dassa E, Bouige P: The ABC of ABCs: a phylogenetic and functional classification of $A B C$ systems in living organisms. Res Microbiol 200I, I 52:2 I I-229.

6. Durbin R, Eddy SR, Krogh A, Mitchison G: Biological sequence analysis. Cambridge University Press, Cambridge, UK; 1998.

7. Edgar R: MUSCLE: a multiple sequence alignment method with reduced time and space complexity. BMC Bioinformatics 2004, 5: II3.

8. Edgar R: MUSCLE: Multiple sequence alignment with high score accuracy and high throughput. Nuc Acids Res 2004, 32: $1792-1797$.

9. Felsenstein J: Confidence limits on phylogenies: an approach using the bootstrap. Evolution 1985, 39:783-791.

10. Felsenstein J: Phylogenies from molecular sequences: inference and reliability. Ann Rev Genet 1988, 22:52 I-565.

II. Felsenstein J: Inferring Phylogenies. Sinauer Associates, Sunderland, MA, USA; 2003.

12. Heringa J: Detection of internal repeats: how common are they? Curr Opin Struc Biol 1998, 8:338-345.

13. Katoh K, Kuma K, Toh H, Miyata T: MAFFT version 5: improvement in accuracy of multiple sequence alignment. Nuc Acids Research 2005, 33:5। I-518.

14. Katoh K, Misawa K, Kuma K, Miyata T: MAFFT: a novel method for rapid multiple sequence alignment based on fast fourier transform. Nuc Acids Research 2002, 30:3059-3066.

15. Köster W: ABC transporter-mediated uptake of iron, siderophores, heme, and vitamin $\mathbf{B}_{12}$. Res Microbiol 1999, | 52:29|-301.

16. Lassmann T, Sonnhammer EL: Quality assessment of multiple alignment programs. FEBS Letters 2002, 529:I 26-I30.

17. Lenhof H-P, Morgenstern B, Reinert K: An exact solution for the segment-to-segment multiple sequence alignment problem. Bioinformatics 1999, 15:203-210. 
18. Linton KJ, Higgins CF: The escheriacoli ATP-binding cassette (ABC). Molecular Microbiology 1998, 28:5-13.

19. Matsusaki S, Watanabe H, Oshima T, Kanaya S, Mori H: Prediction of target substrates of transporters in escherichia coli. Genome Informatics 2002, 13:394-395.

20. Morgenstern B: DIALIGN 2: improvement of the segment-tosegment approach to multiple sequence alignment. Bioinformatics 1999, 15:211-218.

21. Morgenstern B: A space-efficient algorithm for aligning large genomic sequences. Bioinformatics 2000, 16:948-949.

22. Morgenstern B: A simple and space-efficient fragment-chaining algorithm for alignment of DNA and protein sequences. Applied Mathematics Letters 2002, I5: I I-16.

23. Morgenstern B: DIALIGN: Multiple DNA and protein sequence alignment at BiBiServ. Nucleic Acids Research 2004, 32:W33-W36

24. Morgenstern B, Dress A, Werner T: Multiple DNA and protein sequence alignment based on segment-to-segment comparison. Proc Natl Acad Sci USA 1996, 93: I 2098-12 103.

25. Morgenstern B, Goel S, Sczyrba A, Dress A: AltAVisT: a WWW server for comparison of alternative multiple sequence alignments. Bioinformatics 2003, 19:425-426.

26. Morgenstern B, Prohaska SJ, Pöhler D, Stadler PF: Multiple sequence alignment with user-defined anchor points. Algorithms for Molecular Biology 2006, I:6.

27. Morgenstern B, Werner N, Prohaska SJ, Schneider RSI, Subramanian $A R$, Stadler PF, Weyer-Menkhoff J: Multiple sequence alignment with user-defined constraints at GOBICS. Bioinformatics 2005, 2I:127|-I273.

28. Needleman SB, Wunsch CD: A general method applicable to the search for similarities in the amino acid sequence of two proteins. J Mol Biol 1970, 48:443-453.

29. Saitou N, Nei M: The neighbor-joining method: a new method for reconstructing phylogenetic trees. Mol Biol Evol 1987, 4(4):406-425.

30. Saurin W, Hofnung M, Dassa E: Getting in or out: Early segregation between importers and exporters in the evolution of atp-binding cassette (ABC) transporters. J Mol Evol 1999, 48:22-41.

31. Schneider E, Hunke S: ATP-binding cassette (ABC)-transport systems: Functional and structural aspects of the ATP-binding subunit of an ABC-transporter. FEMS Microbiol Rev 1998, 22: $1-20$.

32. Subramanian AR, Kaufmann M, Morgenstern B: DIALIGN-TX: greedy and progressive approaches for the segment-based multiple sequence alignment. Algorithms for Molecular Biology 2008, 3:6.

33. Subramanian AR, Weyer-Menkhoff J, Kaufmann M, Morgenstern B: DIALIGN-T: An improved algorithm for segment-based multiple sequence alignment. BMC Bioinformatics 2005, 6:66.

34. Swofford D, Olsen G: Phylogeny reconstruction. In Molecular Systematics Edited by: Hillis D, Moritz C. Sinauer Associates; 1990:407-5II.

35. Thompson JD, Higgins DG, Gibson TJ: CLUSTAL W: improving the sensitivity of progressive multiple sequence alignment through sequence weighting, position-specific gap penalties and weight matrix choice. Nucleic Acids Research 1994, 22:4673-4680.

36. Thompson JD, Plewniak F, Poch O: A comprehensive comparison of protein sequence alignment programs. Nucleic Acids Research 1999, 27:2682-2690.
Publish with Bio Med Central and every scientist can read your work free of charge

"BioMed Central will be the most significant development for disseminating the results of biomedical research in our lifetime. "

Sir Paul Nurse, Cancer Research UK

Your research papers will be:

- available free of charge to the entire biomedical community

- peer reviewed and published immediately upon acceptance

- cited in PubMed and archived on PubMed Central

- yours - you keep the copyright

Submit your manuscript here:

http://www.biomedcentral.com/info/publishing_adv.asp
BioMedcentral 